Please cite as: Lopes, P.; Leandro, J.; Carvalho, R.F.; Páscoa, P. and Martins, R. 2015. Numerical and experimental investigation of a gully under surcharge conditions. Urban Water Journal, 12 (6), 468-476, DOI: 10.1080/1573062X.2013.831916.

\title{
Numerical and experimental investigation of a gully under surcharge conditions
}

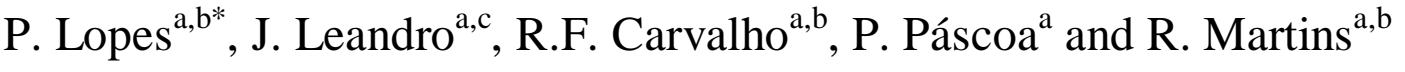 \\ ${ }^{a}$ Institute of Marine Research - Centre of marine and environmental sciences, IMAR- \\ CMA, Department of Life Sciences, Coimbra, Portugal. \\ ${ }^{b}$ Civil Engineering Department, University of Coimbra, Coimbra, Portugal. \\ ${ }^{c}$ Institute of Hydrology, Water Management and Environmental Techniques, Ruhr- \\ University Bochum, Germany.
}

\begin{abstract}
This paper deals with numerical and experimental results of a gully when the sewer system becomes pressurized. These results are useful for the calibration and validation of the linking elements found in Dual Drainage (DD) models. The experimental results were obtained in the MLE (Multiple-LinkingElement) experimental installation that allows the simulation of full surcharge flow through a gully. The installation consists of a $8 \mathrm{~m}$ long and $0.5 \mathrm{~m}$ wide channel, fitted with a $0.6 \times 0.3 \times 0.3 \mathrm{~m}$ gully with a $80 \mathrm{~mm}$ diameter pipe inlet at the bottom. The numerical results were obtained using a three-dimensional structured mesh simulated in the OpenFOAM ${ }^{\mathrm{TM}}$ Toolbox. The results characterization focuses mainly on the jet area, whereby pressure-flow relations were derived for this specific gully. The good agreement found between numerical and experimental results, allowed the extrapolation to larger flow rates.
\end{abstract}

Keywords: urban drainage models, gully, surcharge flow, CFD, experimental data

\section{Introduction}

During extreme rainfall events urban drainage systems may become pressurized, and the flow may surcharge through the gullies and manholes. This phenomenon is commonly called "reverse flow" (Lopes et al., 2012). In extreme cases it may cause

\footnotetext{
*Corresponding author. E-mail: pmlopes@ @student.dec.uc.pt
} 
Please cite as: Lopes, P.; Leandro, J.; Carvalho, R.F.; Páscoa, P. and Martins, R. 2015. Numerical and experimental investigation of a gully under surcharge conditions. Urban Water Journal, 12 (6), 468-476, DOI: 10.1080/1573062X.2013.831916.

"urban geysers" and sometimes the projection of the grate that covers the gully. To understand the hydraulics of drainage systems it is therefore important to characterize the hydraulic behaviour of each component of the sewer system.

There are a large variety of models used to simulate the behaviour of flooding in cities. Among those, are the so called Coupled Urban Drainage Models or simply Dual Drainage (DD) models (Leandro et al., 2009). Those are considered the best models to accurately simulate the flows which occur simultaneously on the surface and on the sewer systems. However, DD models fundamental weakness is the existence of few studies where the linking-elements (e.g. manholes or gullies) are actually calibrated.

Experimental and numerical studies in manholes already exist; Guymer et al. (2005) presented some experimental results in surcharged manholes that explored the effects of diameter and surcharge on the solute transport. Stovin et al. (2008) and Bennett et al. (2011) used the previews experimental results to validate a 3D manhole CFD model.

Particular studies on Gullies are fewer than of manholes, despite both having similar costs of experimental installations and computational efforts. The striking difference is the variability of the gullies geometry used in different countries which makes it difficult the definition of a standard efficiency. Gómez \& Russo (2009) obtained experimental equations for the hydraulic efficiency of several continuous transverse grates used in Spain. Romagnoli et al. (2013) studied experimentally drainage flow in the Multiple-Linking-Elements (MLE) experiment. Carvalho et al. (2011) used a 2DV in-house numerical model to study some Portuguese (PT) gullies in drainage conditions and more recently in reverse conditions (Carvalho et al., 2012). Galambos (2012) presented experimental and numerical investigations using 3D Computational Fluid Dynamics (CFD) model to investigate the hydraulic performance 
Please cite as: Lopes, P.; Leandro, J.; Carvalho, R.F.; Páscoa, P. and Martins, R. 2015. Numerical and experimental investigation of a gully under surcharge conditions. Urban Water Journal, 12 (6), 468-476, DOI: 10.1080/1573062X.2013.831916.

of UK gullies during drainage and surcharge conditions. Other studies on Portuguese gullies using 3D CFD OpenFOAM ${ }^{\mathrm{TM}}$ are performed in Martins et al. (2012) and Lopes et al. (2012).

The work presented herein intents to be a step forwards in understanding the hydraulic behaviour of the Portuguese gullies in reverse conditions, which can be useful for the calibration of linking elements DD models. The experimental installation MLE constructed at the Laboratory of Hydraulics and Water Resources of University of Coimbra is able to simulate flows in the range of 2 to $61 / \mathrm{s}$ at the inlet. The numerical results are obtained using the OpenFOAM ${ }^{\mathrm{TM}}$ open-source toolbox.

The paper starts by presenting the experimental installation and numerical Model used, followed by the methodology used for comparing the numerical and experimental studies developed for verifying the accuracy of the numerical model, particularly in the area close to the jet. The paper then presents the extrapolation to larger discharge rates by using the calibrated numerical model while the behaviour of the flow inside the gully box is analysed.

\section{Experimental Setup}

The physical tests were carried out in an existent full scale experimental gully inside a multipurpose channel at the Hydraulic Laboratory of University of Coimbra where the channel flow, the pumps regime and the valves are electronically controlled by a SCADA system (Supervision, Control And Data Acquisition). The experimental facility is composed by an acrylic platform with $1 \%$ slope, $8 \mathrm{~m}$ long and $0.5 \mathrm{~m}$ wide and an acrylic gully box with dimensions $0.6 \mathrm{~m}$ long $0.3 \mathrm{~m}$ deep and $0.3 \mathrm{~m}$ wide (Figure 1 ). In order to simulate the surcharge conditions, the gully bottom is connected to a small reservoir by a $0.08 \mathrm{~m}$ internal diameter PVC opaque pipe. The water level in this 
Please cite as: Lopes, P.; Leandro, J.; Carvalho, R.F.; Páscoa, P. and Martins, R. 2015. Numerical and experimental investigation of a gully under surcharge conditions. Urban Water Journal, 12 (6), 468-476, DOI: 10.1080/1573062X.2013.831916.

reservoir can provide the control of the static pressure and the verification of the steady state flow. The experiment downstream outlet is a free outfall. This structure can be used to replicate a range of discharges from 2 to 6 1/s.

The photographs of the flow were taken using digital Sony Alpha DSLR-A350 camera with 14.2 MP with a lens DT18-70 mm F3.5-5.6. The camera was supported by a tripod during all the experiments fixed in a specific place. To provide the bubbles illumination, a reflector Photoflex SilverDome NXT with 1000W light was used. The instantaneous velocity data set were collected using a Nortek $\mathrm{AS}^{\circledR} 10 \mathrm{MHz}$ Acoustic Doppler Velocimeter (ADV).

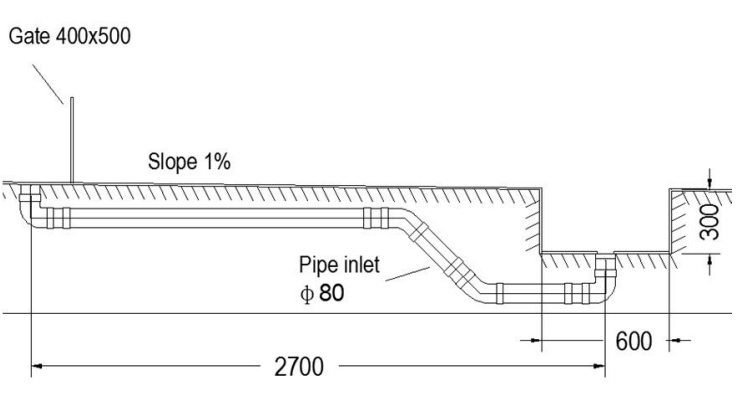

Figure 1a - Sketch of the experimental installation used for the study of the reverse flow $[\mathrm{mm}]$.

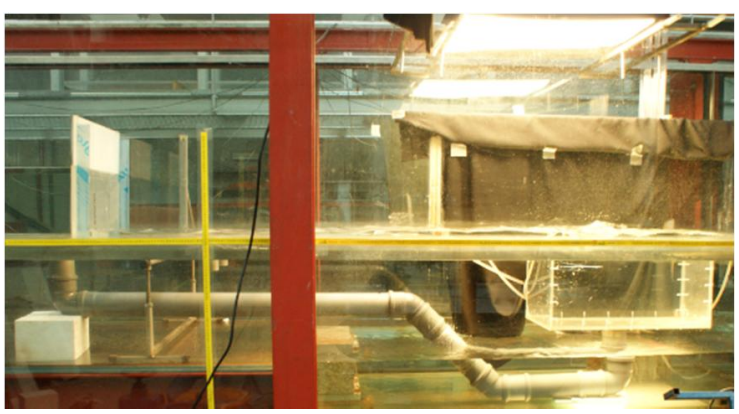

Figure $1 \mathrm{~b}-$ Photograph of the experimental installation.

\section{Numerical Simulations}

Solutions are obtained using OpenFOAM ${ }^{\mathrm{TM}}$ v1.7.1 with the solver interFOAM (Ubbink, 1997) and PISO algorithm (Pressure Implicit Splitting of Operators) (Issa, 1985) used for interactive computation of unsteady incompressible flows. The solver interFOAM is able to reproduce the multiphase flows with VOF (Volume-Of-Fluid) method (Hirt and Nichols, 1981) where a transport equation is able to determine the relative volume fraction of the two phases ("alpha1" in OpenFOAM nomenclature) in 
Please cite as: Lopes, P.; Leandro, J.; Carvalho, R.F.; Páscoa, P. and Martins, R. 2015. Numerical and experimental investigation of a gully under surcharge conditions. Urban Water Journal, 12 (6), 468-476, DOI: 10.1080/1573062X.2013.831916.

each computational cell.

The Smagorinsky LES (Large Eddy Simulation) model (Smagorinsky, 1963) was used. It can reproduce similar results with less computational effort than DNS (Direct Navier Stokes), solving only the large scales of turbulence and approximate the smalls ones using simplified methods, similar to RANS (Reynolds-Average Navier Stokes), the SGS models (Sub-Grid Scale). In this model, the eddy-viscosity ( $\mu_{\mathrm{t}}$ or $\mu_{\mathrm{SGS}}$ ) is modelled using a constant Cs relatable with the constants $\mathrm{C} \varepsilon$ and $\mathrm{Ck}$. The pre-defined values in OpenFOAM are $\mathrm{C} \varepsilon=1.05, \mathrm{Ck}=0.07$ and $\mathrm{Cs}=0.13$ (Damián and Nigro, 2010).

\section{Methodology}

\section{Experimental proceedings}

Three different tests were carried out with sewer discharges of 4,5 and 6 1/s. These flows are controlled upstream by the SCADA system and through the heights in the reservoir. Nevertheless, as these discharges were near the low limit range for SCADA, they were confirmed by the volumetric method. Table1 summarize the tests name (Qx), flow rates $(Q)$, internal diameter of the pipe inlet (D), velocity at the inlet (U), Reynolds numbers (Re) and Froude numbers (Fr).

Table 1 - Summary of the discharge conditions performed in the experimental installation. Q - Flow at inlet (overflow), D - Internal diameter of pipe inlet, U Velocity at inlet, Re - Reynolds Number, Fr - Froude Number.

\begin{tabular}{cccccc}
\hline Test Name & $\mathbf{Q}(\mathbf{l} / \mathbf{s})$ & $\mathbf{D}(\mathbf{m})$ & $\mathbf{U}(\mathbf{m} / \mathbf{s})$ & $\operatorname{Re}(-)$ & $\mathbf{F r}(-)$ \\
\hline $\mathbf{Q 4}$ & 4 & 0.08 & 0.796 & $6.37 \times 10^{4}$ & 0.90 \\
$\mathbf{Q 5}$ & 5 & 0.08 & 0.995 & $7.96 \times 10^{4}$ & 1.12 \\
$\mathbf{Q 6}$ & 6 & 0.08 & 1.194 & $9.55 \times 10^{4}$ & 1.35 \\
\hline
\end{tabular}

Each flow was filmed and photographed 10 times and the most representative photograph was chosen to compare visually with the numerical results. Furthermore a 
Please cite as: Lopes, P.; Leandro, J.; Carvalho, R.F.; Páscoa, P. and Martins, R. 2015. Numerical and experimental investigation of a gully under surcharge conditions. Urban Water Journal, 12 (6), 468-476, DOI: 10.1080/1573062X.2013.831916.

Computational Vision Model in Simulink ${ }^{\mathrm{TM}}$ developed by Roque (2011) was used to find the average contour for the free surface using the entire film. This model is based in image treatment and segmentation techniques to find at each instant the two largest lines within the domain for a given spatial threshold. One of those lines represents the channel bottom and the other the free surface. The distance between the midpoints of those lines is the flow depth. The acquisition rate used in the model was set to $30 \mathrm{fps}$.

Measurements of the velocity of the jet were obtained using an ADV probe. The low ADV correlations obtained inside the jet prevented the use of such points, therefore, only the points in the vicinity of the jet were used. In total, 19 points were measured in two vertical lines with $0.03 \mathrm{~m}$ spacing (Figure 2). The frequency and sampling period were set to $1 \mathrm{~Hz}$ and $180 \mathrm{~s}$ respectively.

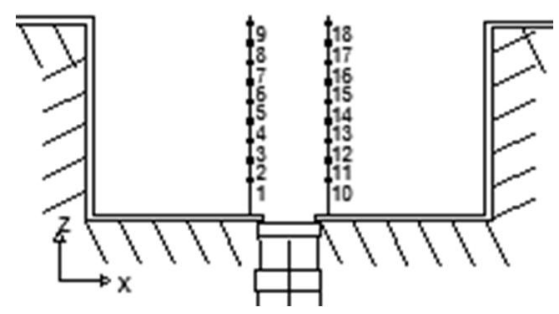

Figure 2 - Points measured at the left (points 1 to 9) and right side of the gully (points 10 to 18 ).

\section{Computational Meshes}

To accurately replicate the physical experiment, some adjustments were made in the mesh. A previous study from the Authors showed good results with the application of a sudden enlargement and a curve at the gully inlet to reproduce the head losses in the pipe and the real trajectory of the velocity vectors at the inlet, respectively (Figure 3) (Lopes et al., 2012). 
Please cite as: Lopes, P.; Leandro, J.; Carvalho, R.F.; Páscoa, P. and Martins, R. 2015. Numerical and experimental investigation of a gully under surcharge conditions. Urban Water Journal, 12 (6), 468-476, DOI: 10.1080/1573062X.2013.831916.

The mesh used is regular and non-regular with grid spacing between $0.01 \mathrm{~m}$ and $0.04 \mathrm{~m}$, making a total of 278855 points. The finer mesh is applied to the gully box whereas the coarser mesh is applied to the main channel when the vortex characterizations are not so important. The mesh was generated using the blockMesh utility available in the OpenFOAM ${ }^{\mathrm{TM}}$ toolbox. This kind of mesh is harder to generate comparatively to the use of CAD software's (e.g. SALOME platform or GMSH), but the well-structured grid contribute efficiently to the control of the grid space and for the decreasing of the computational time-step.

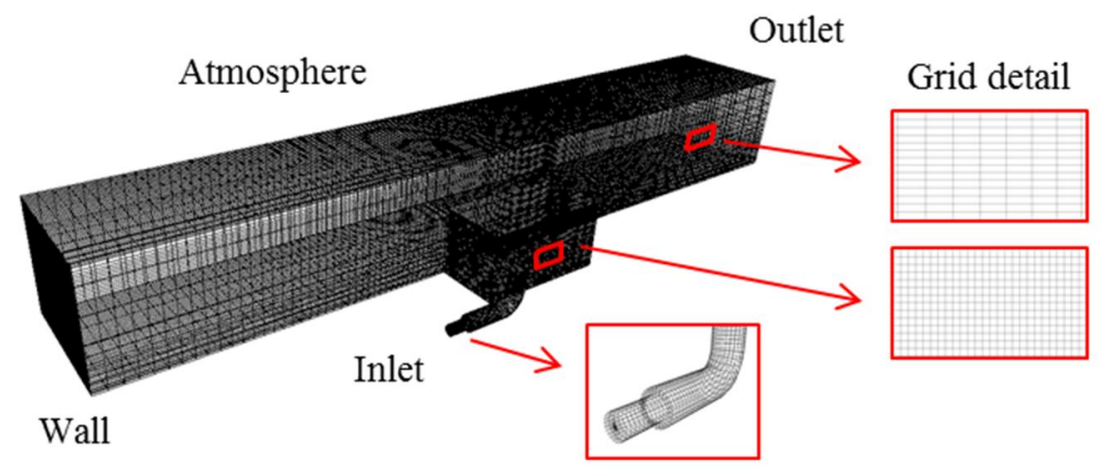

Figure 3 - 3D view of mesh, grid detail and boundary conditions used in the numerical model OpenFOAM for studying reverse flow in the gully.

\section{Boundary Conditions}

Four types of boundary conditions were used in the model. The parameterizations and characteristics depending on their functional propose: The inlet (end of pipe) only allows flow in at a fixed velocity; the outlet is a boundary where the fluids exits the domain (right side of the channel), where the relative pressure is fixed to 0 ; in the atmosphere the air can make exchanges with the outside and the relative pressure is set to 0 and the wall have the condition of no slip and therefore the velocity is set to 0 . When one parameter is stipulated the other boundary parameters are calculated by OpenFOAM $^{\mathrm{TM}}$. 
Please cite as: Lopes, P.; Leandro, J.; Carvalho, R.F.; Páscoa, P. and Martins, R. 2015. Numerical and experimental investigation of a gully under surcharge conditions. Urban Water Journal, 12 (6), 468-476, DOI: 10.1080/1573062X.2013.831916.

\section{Computational simulations}

All the numerical results were obtained after 15 seconds of simulation. The Authors experience in similar structures and computational simulations showed that 10 or 15 seconds of simulation were sufficient to reach the steady state (Carvalho et al., 2012; Martins et al., 2012; Lopes et al., 2012). The average results obtained are related to the last 5 seconds of simulation.

The computational simulations were performed using turbulence closure. The Smagorinsky LES model was chosen because it shows good consistency comparatively to experimental results (Gong and Tanner, 2009). The two parameters Ce and Ck need however to be adjusted to each case (Damián and Nigro, 2010). Several values were tested around the standard values proposed in OpenFOAM ${ }^{\mathrm{TM}}$ Toolbox, $\mathrm{C} \varepsilon=1.05$ and $\mathrm{Ck}=0.07$ for the simulation $\mathrm{Q} 5$. In total, five simulations with different combinations were computed ( $\mathrm{C} 1$ to $\mathrm{C} 5)$. The average relative errors (ARE) between numerical and experimental results for the velocity near the centre of the gully are showed in Table 2 and errors around $30 \%$ were found. However, these errors are acceptable in the vicinity of jets because of the large temporal variation of the velocity profile as attested by other authors (Hussein et al., 1994). Nevertheless the selection of the parameters C $\varepsilon=1.05$ and $\mathrm{Ck}=0.03$ (combination C3) was based on the ARE (Table 2). Figure 3 shows the experimental and the numerical data for flow heights above the gully.

Table 2 - Average Relative Errors (ARE) for numerical and experimental velocities at left (AREl) and at right (AREr) points of gully (see Error! Reference source not found.) for the five set of LES coefficients tested.

\begin{tabular}{lccccc}
\hline & $\mathrm{C} \varepsilon$ & $\mathrm{Ck}$ & AREl & AREr & ARE \\
\hline $\mathrm{C} 1$ & 1.05 & 0.07 & $35 \%$ & $30 \%$ & $33 \%$ \\
$\mathrm{C} 2$ & 1.05 & 0.055 & $35 \%$ & $42 \%$ & $38 \%$ \\
$\mathbf{C 3}$ & $\mathbf{1 . 0 5}$ & $\mathbf{0 . 0 3}$ & $\mathbf{3 1 \%}$ & $\mathbf{2 7 \%}$ & $\mathbf{2 9 \%}$ \\
$\mathrm{C} 4$ & 1.15 & 0.07 & $41 \%$ & $21 \%$ & $31 \%$ \\
$\mathrm{C} 5$ & 1.25 & 0.07 & $25 \%$ & $37 \%$ & $31 \%$ \\
\hline
\end{tabular}


Please cite as: Lopes, P.; Leandro, J.; Carvalho, R.F.; Páscoa, P. and Martins, R. 2015. Numerical and experimental investigation of a gully under surcharge conditions. Urban Water Journal, 12 (6), 468-476, DOI: 10.1080/1573062X.2013.831916.

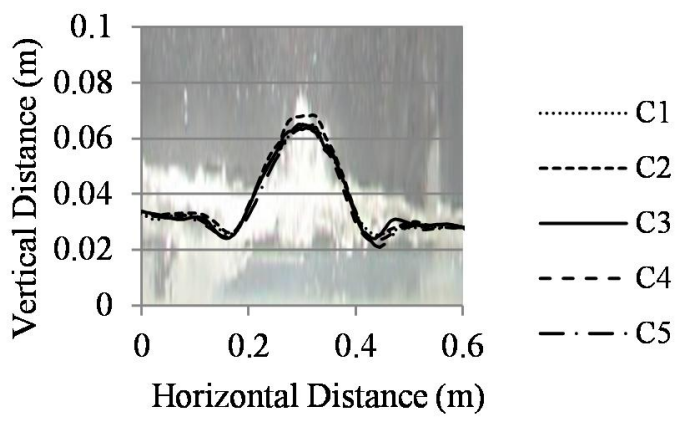

Figure 4 - Heights of the flow for different set of LES parameters ( $\mathrm{C} \varepsilon$ and $\mathrm{Ck}$ ) using the simulation with $5 \mathrm{l} / \mathrm{s}$ at the inlet (Q5).

\section{Results}

Figure 5 shows the numerical average contour in the central section of the gully. The computational simulations are compared with the representative photograph of each flow and the average contour of the jet extracted from the Computational Vision Model (Roque, 2011).

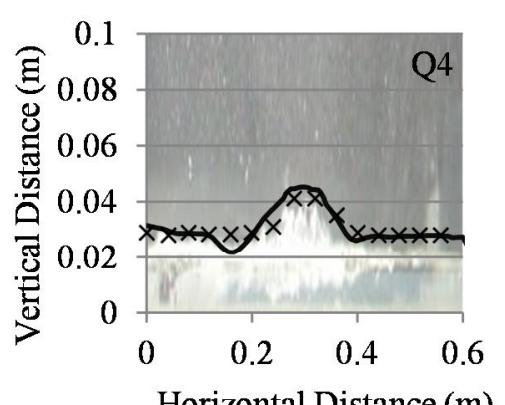

Horizontal Distance (m)
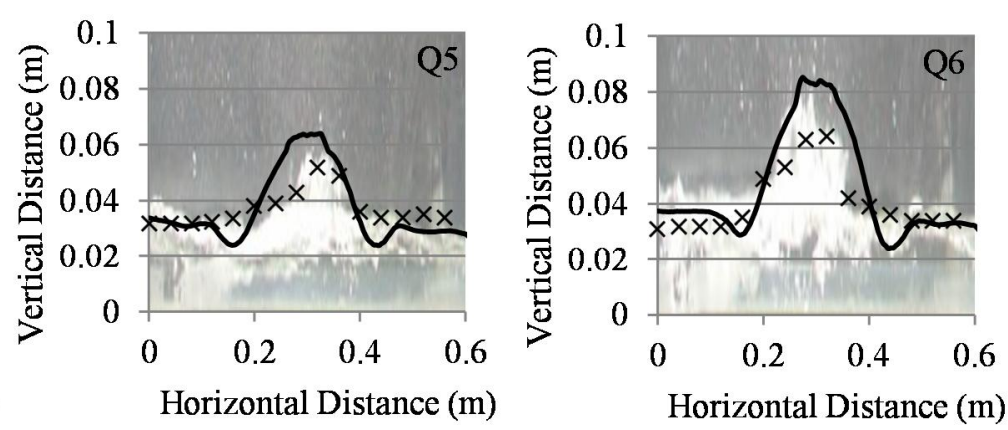

- OF Numerical Model $\quad \times$ Computational Vision Model

Figure 5 - Average contour obtained with OpenFOAM numerical model compared with photographs in background and a Computational Vision Model (Roque, 2011).

After calibration of the jet through the experimental photographs, new numerical simulations are computed for flows with 8 and 10 1/s at the inlet (those simulations are symbolized with *). Figure $6 \mathrm{a}$ and Figure $6 \mathrm{~b}$ shows respectively the total pressure $(\mathrm{Pt})$ 
Please cite as: Lopes, P.; Leandro, J.; Carvalho, R.F.; Páscoa, P. and Martins, R. 2015. Numerical and experimental investigation of a gully under surcharge conditions. Urban Water Journal, 12 (6), 468-476, DOI: 10.1080/1573062X.2013.831916.

and dynamic pressure $(\mathrm{Pd})$ at gully bottom in the center profile of the gully for the simulations Q4, Q5, Q6, Q8 and Q10 1/s. The total pressure is the sum of static pressure with dynamic pressure $(\mathrm{Pt}=\mathrm{P}+\mathrm{Pd})$.

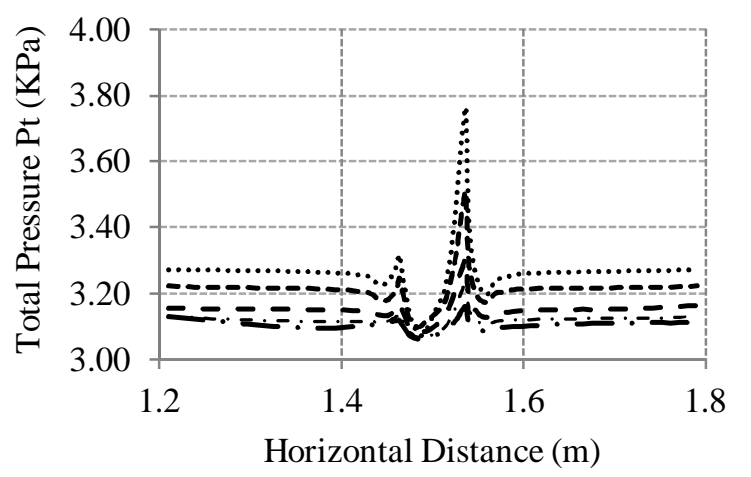

$-\cdot-\mathrm{Q} 4 \quad-\cdot-\cdot \mathrm{Q} 5 \quad---\mathrm{Q} 6$

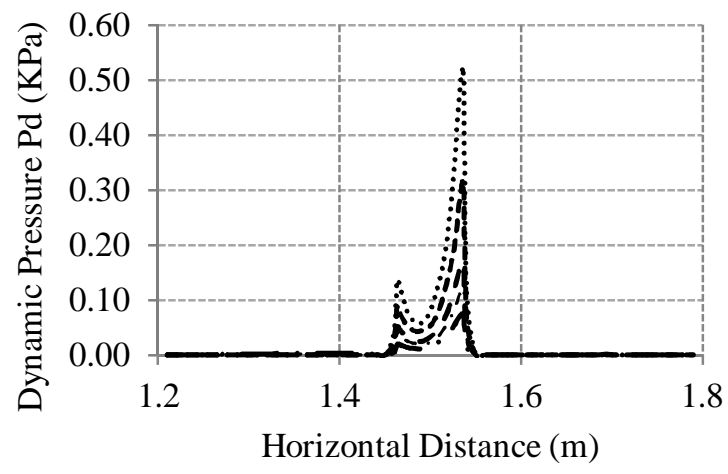

Q10*

Figure $6 \mathrm{a}-$ Total pressure $(\mathrm{Pt})$ at the gully Figure $6 \mathrm{~b}$ - Dynamic pressure $(\mathrm{Pd})$ at the bottom in the centre profile. gully bottom in the centre profile.

Figure 7a shows the maximum heights of the jet $(\mathrm{hJ})$ and the flow depths at the left $(\mathrm{hL})$ and the right side $(\mathrm{hR})$ of the gully. Figure $7 \mathrm{~b}$ shows the average total pressure $(\mathrm{Pt})$ and average dynamic pressure $(\mathrm{Pd})$ at gully bottom in the centre $(\mathrm{C})$, left $(\mathrm{L})$ and right side (R) of the gully. The adjustments to the best trend lines are made herein and the relations for h-Q (flow height - sewer overflow), Pt-Q (total pressure - sewer overflow) and Pd-Q (dynamic pressure - sewer overflow) are shown. 
Please cite as: Lopes, P.; Leandro, J.; Carvalho, R.F.; Páscoa, P. and Martins, R. 2015. Numerical and experimental investigation of a gully under surcharge conditions. Urban Water Journal, 12 (6), 468-476, DOI: 10.1080/1573062X.2013.831916.

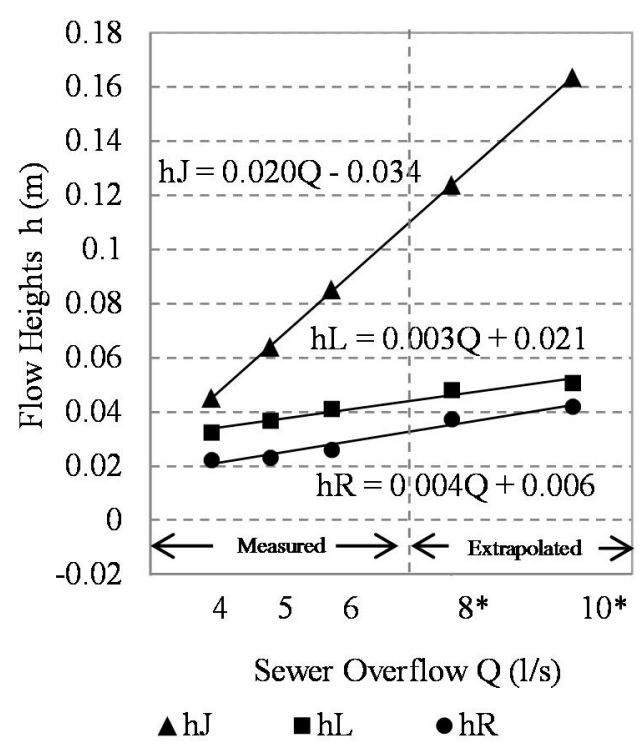

Figure $7 \mathrm{a}-$ Flow heights in top of the gully. The triangles represent the maximum height of the jet $(\mathrm{hJ})$, the squares represent the height at left side of the jet $(\mathrm{hL})$, while the circles represents the height at right side $(\mathrm{hR})$. The relations $\mathrm{h}-\mathrm{Q}$ are shown in the figure.

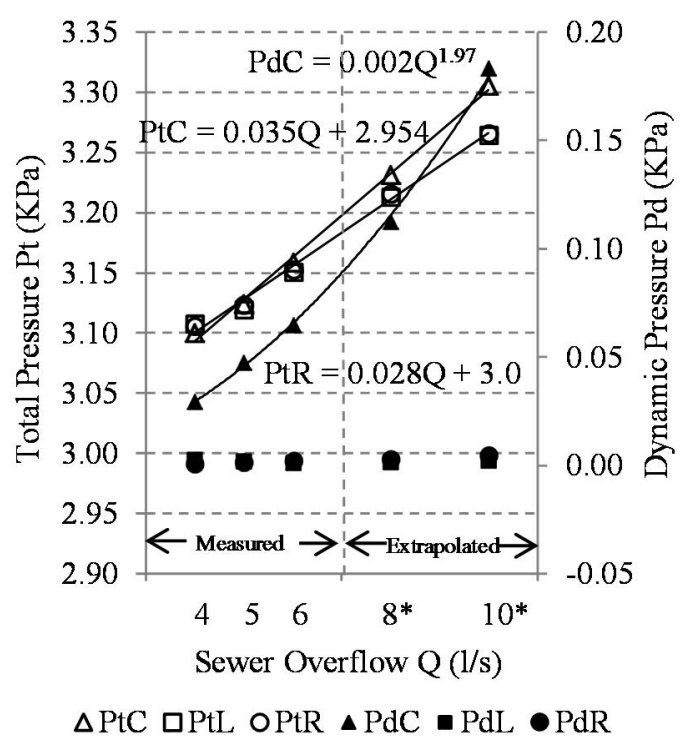

Figure $7 b-$ Average total pressure $(\mathrm{Pt})$ and average dynamic pressure $(\mathrm{Pd})$ at gully bottom. The triangles represent the average pressure at the inlet (C), the squares represent the average pressure at left side of gully bottom (L), while the circles represents average pressure at right side of gully bottom (R). The relations Pt$\mathrm{Q}$ and $\mathrm{Pd}-\mathrm{Q}$ are shown in the figure.

Figure 8 shows the average stream lines of the gully in the middle longitudinal section. Figure 9 shows the average velocity fields in the middle longitudinal section of the gully obtained from the numerical model using a grey scale in the range 0 to $3.3 \mathrm{~m} / \mathrm{s}$.

Q4
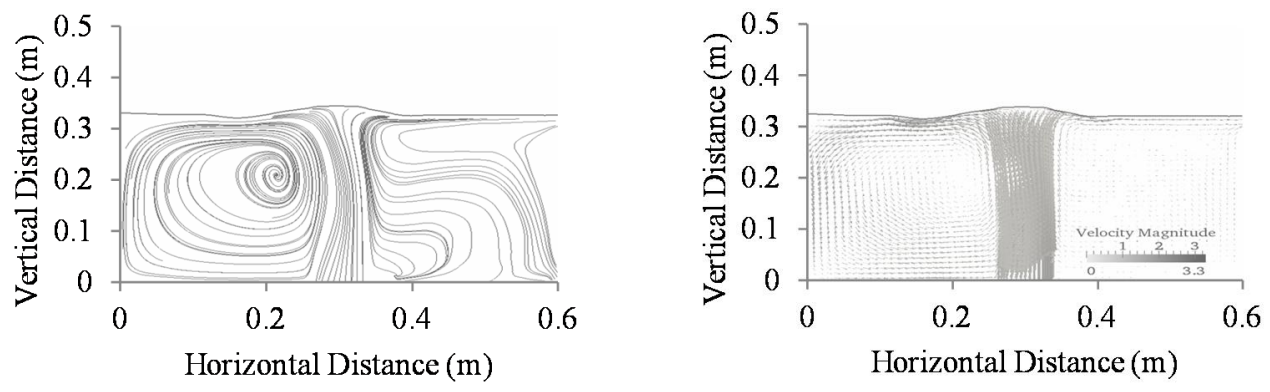
Please cite as: Lopes, P.; Leandro, J.; Carvalho, R.F.; Páscoa, P. and Martins, R. 2015. Numerical and experimental investigation of a gully under surcharge conditions. Urban Water Journal, 12 (6), 468-476, DOI: 10.1080/1573062X.2013.831916.

Q5

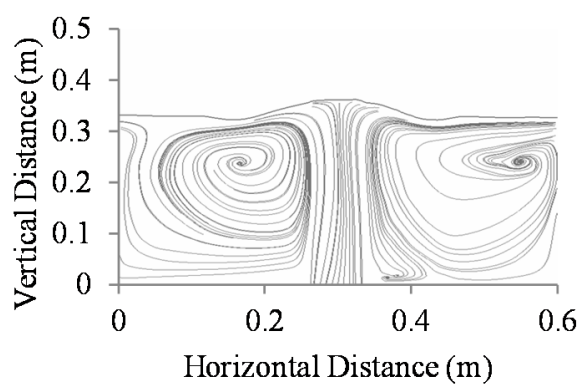

Q6

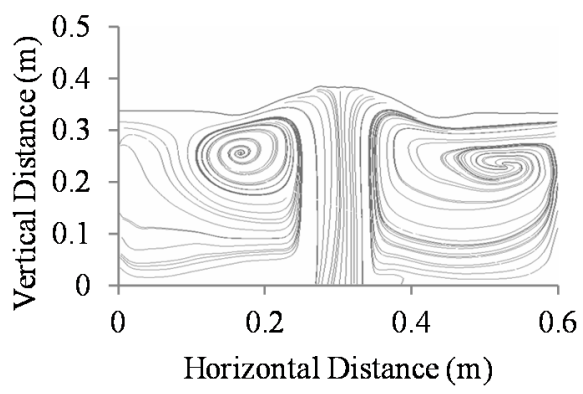

Q8*

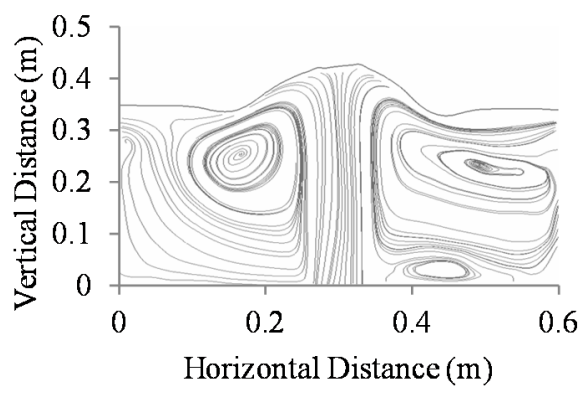

Q10*

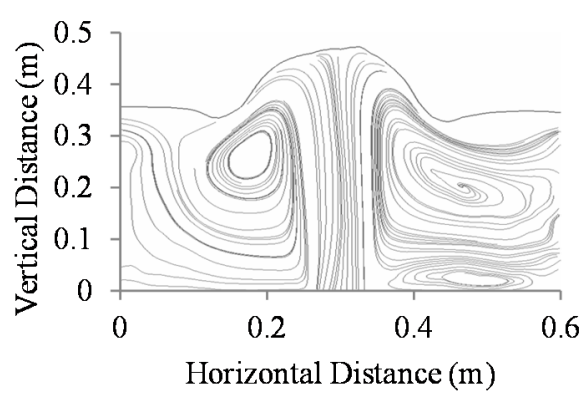

Figure 8 - Stream lines obtained with Figure 9 - Velocity fields obtained the numerical model in the middle with the numerical model in the longitudinal section of the gully. middle longitudinal section of the *Extrapolated discharges
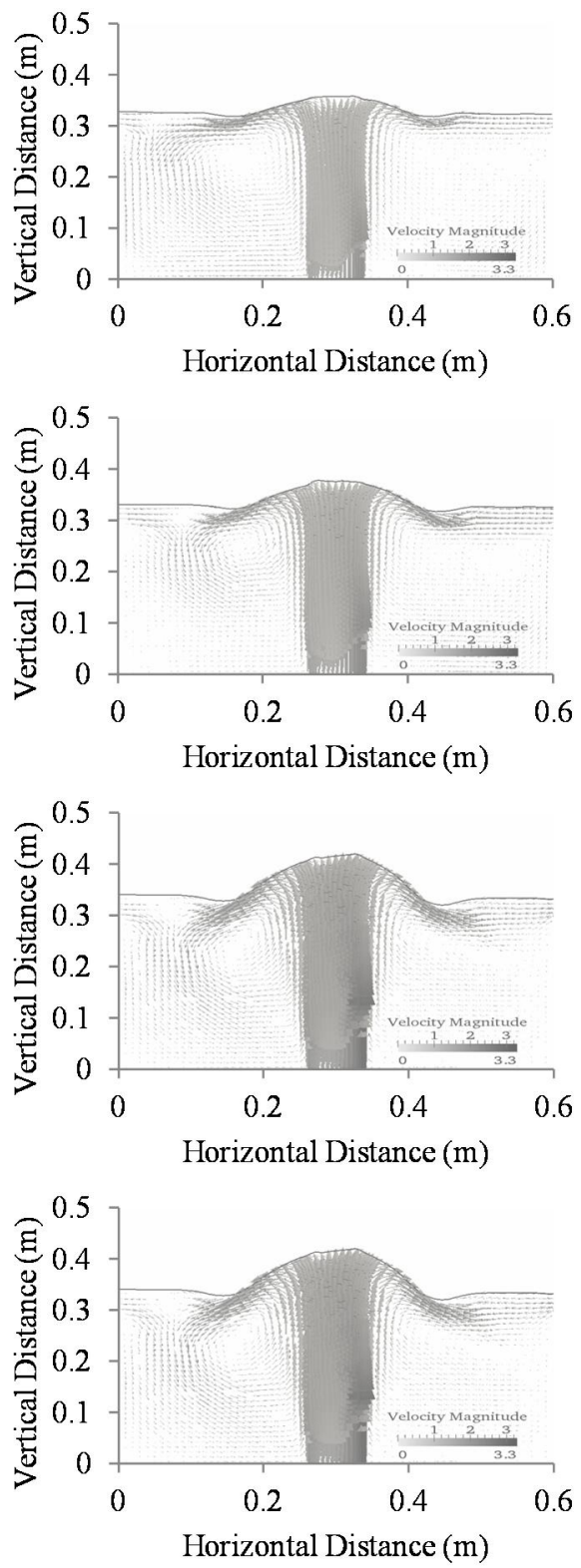
gully. *Extrapolated discharges

Figure 11 represents the temporal velocities in a point centred in the top of the gully (Figure 10) for the simulation Q6. Analogous behaviour was found for the remaining simulations. For the sake of simplicity they are not presented in this paper. 
Please cite as: Lopes, P.; Leandro, J.; Carvalho, R.F.; Páscoa, P. and Martins, R. 2015. Numerical and experimental investigation of a gully under surcharge conditions. Urban Water Journal, 12 (6), 468-476, DOI: 10.1080/1573062X.2013.831916.

Figure 12 shows the application of Fast Fourier Transforms (FFT) to the theta angle and the vector $\mathrm{Vz}$ for the simulation Q6. The FFT is used to obtain the dominant frequency of the signals or periodic structures in time series data.

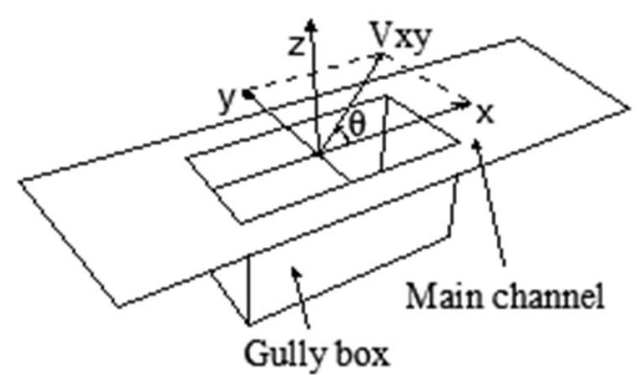

Figure 10 - Sketch of centre point in the top of the gully and theta angle used for the study of the behaviour of the jet.
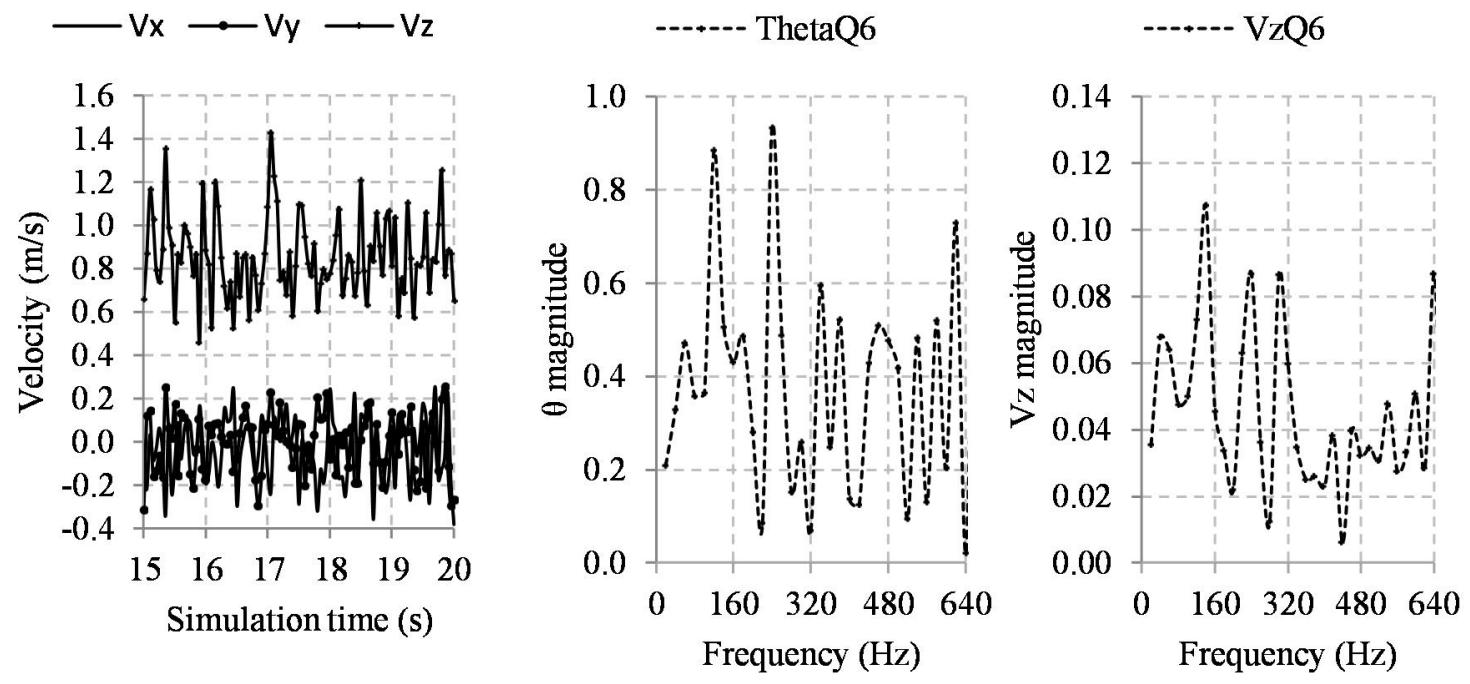

Figure 11 - Velocities at Figure 12 - FFT analysis for the theta angle and directions $\mathrm{x}, \mathrm{y}$ and $\mathrm{z}$ in the velocity Vz using Data Analysis Package using Q6. center point on the top of the gully for Q6.

Figure 13a represents a histogram of frequencies for the direction of the vector Vxy through a theta angle (in radians), schematically represented in the Figure 10. If the 
Please cite as: Lopes, P.; Leandro, J.; Carvalho, R.F.; Páscoa, P. and Martins, R. 2015. Numerical and experimental investigation of a gully under surcharge conditions. Urban Water Journal, 12 (6), 468-476, DOI: 10.1080/1573062X.2013.831916.

direction of the vector Vxy is the same of $\mathrm{x}$-axis, the theta angle is equal to zero and evolves anti-clock-wise. The adjustment of maximum $\mathrm{Vz}$ to a linear equation was done herein. Figure $13 \mathrm{~b}$ ) shows a histogram of frequencies of vector $\mathrm{Vz}$ in the centre point in top of gully. Table 3 shows the adjustment to a normal distribution of the Vz histogram using the Sample Kolmogorov-Smirnov (K-S) Test. In this test assuming the null hypothesis the data is better adjusted by a Gaussian distribution.

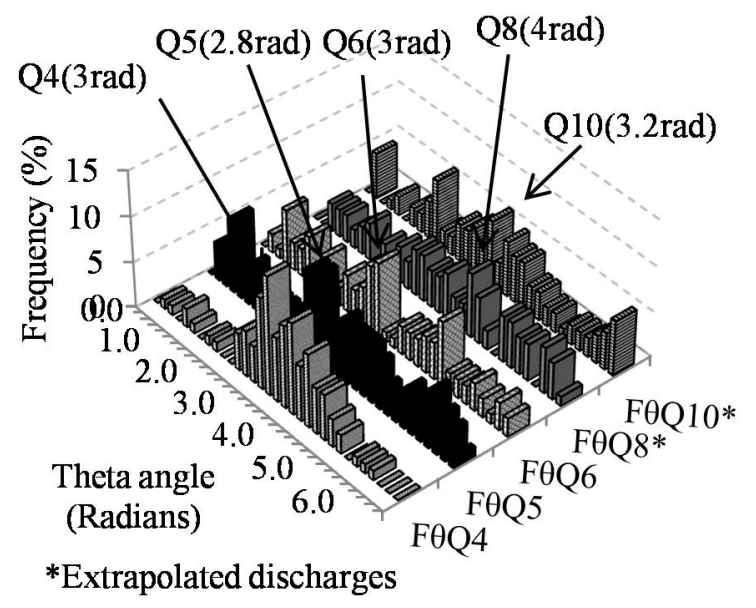

Figure 13a - Histogram of frequencies of the theta angle. The 0 radians means the vector Vxy takes the positive direction of $\mathrm{x}-$ axis. This angle is illustrated schematically in the Figure 10.

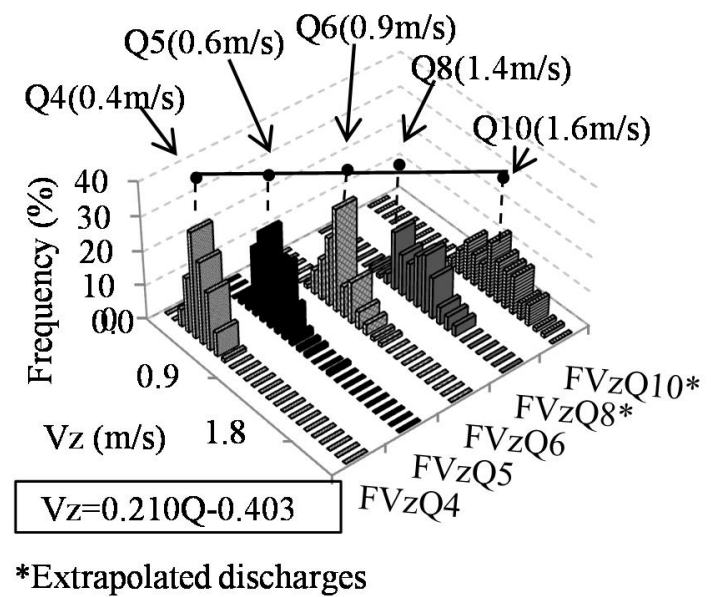

Figure 13b - Histogram of frequencies for the velocity $\mathrm{Vz}$ in the centre point on top of the gully. This point is illustrated schematically in the Figure 10.

Table 3 - Sample Kolmogorov-Smirnov Test for the histograms presented in Figure $13 b$.

\begin{tabular}{|c|c|c|c|c|c|c|}
\hline & & FVzQ4 & FVzQ5 & FVzQ6 & FVzQ8* & FVzQ10* \\
\hline \multicolumn{2}{|c|}{$\operatorname{Mean}(\boldsymbol{\mu})$} & 0.405 & 0.629 & 0.854 & 1.229 & 1.578 \\
\hline \multicolumn{2}{|c|}{ Standard Deviation ( $\sigma$ ) } & 0.130 & 0.182 & 0.188 & 0.235 & 0.281 \\
\hline \multicolumn{2}{|c|}{ p-value of K-S test } & 0.837 & 0.616 & 0.138 & 0.694 & 0.861 \\
\hline \multirow{2}{*}{$\begin{array}{c}95 \% \\
\text { Confidence }\end{array}$} & Min. & 0.150 & 0.272 & 0.486 & 1.148 & 1.481 \\
\hline & Max. & 0.659 & 0.985 & 1.222 & 1.309 & 1.674 \\
\hline
\end{tabular}

*Extrapolated discharges 
Please cite as: Lopes, P.; Leandro, J.; Carvalho, R.F.; Páscoa, P. and Martins, R. 2015. Numerical and experimental investigation of a gully under surcharge conditions. Urban Water Journal, 12 (6), 468-476, DOI: 10.1080/1573062X.2013.831916.

\section{Discussion}

The comparisons of the flow heights in the numerical and the experimental simulations are in good agreement. Figure 5 shows coherent results between numerical average contours, photographs of the experiments, and the free-surface contours captured with the Computational Vision Model.

Figure 6a shows the pressure at the gully bottom in the centre profile. The minimum pressure corresponds to the center of the jet although the maximum values are near the side walls. At the gully sides the total pressure is almost constant and approximately equal to the hydrostatic pressure. The dynamic pressure, showed in Figure $6 \mathrm{~b}$ is almost null near the bottom wall, affected by the lower velocities of the gully eddies, and their contribution to the total pressure can be neglected. Nevertheless, at the inlet, the contribution of the dynamic pressure for the total pressure should be considered. The asymmetry of the jet could be explained by the influence of the pipe curve used in the experiments and eddies' position.

Relations h-Q are presented in Figure 7a whereas relations P-Q and Pd-Q are presented in Figure 7b. The relations h-Q can be approximated using linear equation, while the relations P-Q and Pd-Q use linear and power law adjustment. The average total pressure at left and right side of the gully is coincident and governed with the same equation $(\mathrm{PR}=\mathrm{PL})$. The average dynamic pressure $(\mathrm{Pd})$ in both sides of the jet are near zero, hence the adjustment to trend line was discarded.

The numerical stream lines and vector fields are showed in Figure 8 and Figure 9 , respectively. The surcharge flow is characterized by a strong jet in the center and two large eddies in both sides of the gully. The increasing of the sewer overflow decreases the size of the eddy presented in the left side of the gully and increases the eddy 
Please cite as: Lopes, P.; Leandro, J.; Carvalho, R.F.; Páscoa, P. and Martins, R. 2015. Numerical and experimental investigation of a gully under surcharge conditions. Urban Water Journal, 12 (6), 468-476, DOI: 10.1080/1573062X.2013.831916.

presented on the right side. Three different situations can be defined: 1) for discharge smaller than 4 1/s, the left vortex occupies all the left side of the gully while on the right side, the vortex is negligible; 2) for discharge between 5 and $61 / \mathrm{s}$, one vortex appear near the right wall, while on the left side the vortex size decreases and changing the direction of the jet; 3) for discharge greater than $81 / \mathrm{s}$ the vortex on the right side takes all the right domain and the left side vortex is closer to the jet.

The FFT analysis shows the existence of a dominant frequency of approximately $240 \mathrm{~Hz}$ and $100 \mathrm{~Hz}$ for the theta angle and for the Vz, respectively. The theta angle frequency (Figure 10) shows a maximum value between 2.8 and 4 radians for the five numerical simulations tested (Figure 13a), i.e. in the negative direction of x-axis. This result is more visible for lower discharge and could be explained by the influence of the large eddy in the left side of the jet. The decrease of the left eddy size also decreases the influence on the jet and therefore a greater variability of frequencies is found.

For the velocity $\mathrm{Vz}$ case, the maximum value increases with the discharge (Figure 13b). A linear equation that connects the maximum values of velocity is found and can be used to extrapolate for larger discharges. The adjustment to a normal distribution was done for the Vz histogram with good results and the $95 \%$ confidence interval around the mean values is showed in the Table 3. After analysing the confidence intervals, the extreme values of confidence interval for Q8 and Q10 are closer to the average value than Q4 and Q5. This could indicate less uncertainty in the average values obtained with large discharge, however here we have no experimental data.

\section{Conclusions}

This paper presented numerical and experimental results of surcharge flow in a Gully, 
Please cite as: Lopes, P.; Leandro, J.; Carvalho, R.F.; Páscoa, P. and Martins, R. 2015. Numerical and experimental investigation of a gully under surcharge conditions. Urban Water Journal, 12 (6), 468-476, DOI: 10.1080/1573062X.2013.831916.

especially focussing on the jet characterization and the height it reaches above the gully. The average contour and jet heights were defined for three experimental and five numerical simulations. Average velocities and streamlines at the center profile of gully were also presented.

The following conclusions were obtained: 1) as expected, the height above the gully reached with the jet increases with the increasing of the sewer overflow; 2) two eddies at the gully box were found for the tested discharges, one at each side of the jet; 3) the jet shows one preferential direction for the negative $x$-axis sharpest for lower discharges; 4) the three relations h-Q, three relations $\mathrm{P}-\mathrm{Q}$ and the relation Pd-Q presented can be used to calibrate the linking elements in Drainage Models.

Future studies could be looking into the gully under normal drainage conditions and analysis of other gully structures.

\section{Acknowledgments}

The authors would like to acknowledge the financial support granted by Portuguese Foundation for Science and Technology (FCT) and the COMPETE ("Programa Operacional Temático Factores de Competitividade"), supported by FEDER ("Fundo Europeu de Desenvolvimento Regional") through Project PTDC/AACAMB/101197/2008. The first author developed the research presented in this paper with Doctoral Grant supported by FCT, Grant No. SFRH/BD/85783/2012. The authors also thank Mr. Joaquim Cordeiro da Silva, the laboratory technician, for the help provided during the experiments. 
Please cite as: Lopes, P.; Leandro, J.; Carvalho, R.F.; Páscoa, P. and Martins, R. 2015. Numerical and experimental investigation of a gully under surcharge conditions. Urban Water Journal, 12 (6), 468-476, DOI: 10.1080/1573062X.2013.831916.

\section{Bibliography}

Bennett, P.R., Stovin, V.R., and Guymer, I., 2011. Improved CFD Simulation Approaches for Manhole Mixing Investigations. In: 12th International Conference on Urban Drainage. Porto Alegre. Brazil, pp. 1-8.

Carvalho, R.F., Leandro, J., Martins, R., Abreu, J.M., and De Lima, J.M.L.P., 2011. 2DV numerical modelling of different flows occurring in gullies. In: 12th International Conference on Urban Drainage. Porto Alegre. Brazil, pp. 1-8.

Carvalho, R.F., Leandro, J., Martins, R., and Lopes, P., 2012. Numerical study of the flow behaviour in a gully. In: 4th IAHR International Symposium on Hydraulic Structures. Porto. Portugal, pp. 1-8.

Damián, S.M. and Nigro, N.M., 2010. Comparison of Single Phase Laminar And Large Eddy Simulation (LES) Solvers Using The OpenFOAM Suite. Asociación Argentina de Mecánica Computacional, XXIX, pp. 3721-3740.

Galambos, I., 2012. Improved Understanding of Performance of Local Controls Linking the above and below Ground Components of Urban Flood Flows. PhD Thesis. University of Exeter, UK, pp. 165.

Gong, Y. and Tanner, F.X., 2009. Comparison of RANS and LES Models in the Laminar Limit for a Flow Over a Backward-Facing Step Using OpenFOAM. In: Nineteenth International Multidimensional Engine Modeling Meeting at the SAE Congress. Detroit. Michigan, pp. 1-6.

Gómez, M. and Russo, B., 2009. Hydraulic Efficiency of Continuous Transverse Grates for Paved Areas. Journal of Irrigation and Drainage Engineering, 135 (2), pp. 225.

Guymer, I., Dennis, P., O’Brien, R., and Saiyudthong, C., 2005. Diameter and Surcharge Effects on Solute Transport across Surcharged Manholes. Journal of Hydraulic Engineering, 131 (4), pp. 312-321.

Hirt, C.W. and Nichols, B.D., 1981. Volume of Fluid (VOF) Method for the Dynamics of Free Boundaries. Journal of Computational Physics, 39, pp. 201-225.

Hussein, H.J., Capp, S.P., and George, W.K., 1994. Velocity measurements in a hightReynolds-number, momentum-conserving, axisymmetric, turbulent jet. J. Fluid Mech., 258, pp. 31-75.

Issa, R.I., 1985. Solution of the implicitly discretised fluid flow equations by operatorsplitting. Journal of Computational Physics, 62 (1), pp. 40-65.

Leandro, J., Chen, A., Djordjević, S., and Savić, D.A., 2009. Comparison of 1D/1D and 1D/2D Coupled (Sewer/Surface) Hydraulic Models for Urban Flood Simulation. Journal of Hydraulic Engineering, 135 (6), pp. 495-504. 
Please cite as: Lopes, P.; Leandro, J.; Carvalho, R.F.; Páscoa, P. and Martins, R. 2015.

Numerical and experimental investigation of a gully under surcharge conditions. Urban Water Journal, 12 (6), 468-476, DOI: 10.1080/1573062X.2013.831916.

Lopes, P., Leandro, J., Carvalho, R.F., and Martins, R., 2012. Hydraulic behaviour of a gully under surcharge conditions. In: 9th International Conference on Urban Drainage Modelling. Belgrade. Serbia, pp. 1-10.

Martins, R., Leandro, J., and Carvalho, R.F., 2012. Hydraulic behaviour of a gully: numerical vs . experimental. In: 9th International Conference on Urban Drainage Modelling. Belgrade. Serbia, pp. 1-9.

Romagnoli, M., Carvalho, R.F., and Leandro, J., 2013. Turbulence characterization in a gully with reverse flow. Journal of Hydraulic Engineering-ASCE.

Roque, J.M., 2011. Medição de alturas de água usando visão computacional num modelo Simulink®. MScThesis. University of Coimbra, Portugal. [in Portuguese], pp. 80.

Smagorinsky, J., 1963. General Circulation Experiments with the Primitive Equations. Weather Review, 91 (3), pp. 99-164.

Stovin, V.R., Guymer, I., and Lau, S.D., 2008. Approaches to validating a 3D CFD manhole model. In: 11th International Conference on Urban Drainage. Edinburgh, Scotland, UK, pp. 1-10.

Ubbink, O., 1997. Numerical prediction of two fluid systems with sharp interfaces. PhD Thesis. Imperial College of Science, UK, pp. 69. 